\title{
Radiotherapeutic management of cervical lymph node metastases from an unknown primary site - experiences from a large cohort treated with modern radiation techniques
}

\author{
Tanja Sprave ${ }^{1,2^{*}}$, Alexander Rühle ${ }^{1,2}$, Katharina Hees ${ }^{3}$, Tobias Kalckreuth ${ }^{1,2}$, Vivek Verma ${ }^{4}$, Raluca Stoian? \\ Constantinos Zamboglou ${ }^{1,2}$, Jens Pfeiffer ${ }^{5}$, Roland Laszig ${ }^{5}$, Andreas Knopf ${ }^{5}$, Anca-Ligia Grosu ${ }^{1,2}$ and \\ Nils H. Nicolay ${ }^{1,2^{*}}$
}

\begin{abstract}
Purpose: To analyze management and outcomes following (chemo)radiation therapy in patients with cervical lymph node metastases from an unknown primary site (CCUP) in a large single-center cohort.

Methods: Between 2008 and 2019, 58 patients with CCUP were treated with (chemo)radiation therapy at the University of Freiburg Medical Center and were included in this analysis. Overall survival (OS), locoregional progression-free survival (PFS) and distant metastasis-free survival (DMFS) were calculated using the Kaplan-Meier method. The use of diagnostic procedures and their impact on oncological outcomes was analyzed by Cox regression, and treatment-related toxicities were quantified.

Results: Median follow-up was 29.9 months (range 4.6-121.9). Twenty-one patients (36.2\%) received definitive RT, 35 (60.3\%) underwent adjuvant RT, and 2 (3.4\%) were treated for oligometastatic disease. Concurrent chemotherapy was prescribed in 40 patients (69.0\%). $89.6 \%$ of patients completed the prescribed RT, and $65.0 \%$ completed the prescribed simultaneous chemotherapy. Locoregional recurrence was observed in 7 patients (12.1\%) and distant metastases in 13 cases (22.4\%). OS was 81,1,64.9\% and 56,6\% after 1, 3 and 5 years, respectively. Univariate analysis of age, gender, extracapsular spread, tumor grading, neck dissection, diagnostic utilization of ${ }^{18} \mathrm{~F}$ fluorodeoxyglucose positron-emission tomography and concomitant chemotherapy showed no effect on OS ( $p>$ 0.05 for all), while smoking was significantly associated with decreased survival $(p<0.05)$. There was a trend towards impaired OS for patients with advanced nodal status ( $p N 3)(p=0.07)$. Three patients (5.2\%) experienced grade 3 radiation dermatitis, and 12 (22.4\%) developed grade 3 and 1 (1.7\%) grade 4 mucositis.

(Continued on next page)
\end{abstract}

\footnotetext{
*Correspondence: tanja.sprave@uniklinik-freiburg.de; nils.nicolay@uniklinikfreiburg.de

${ }^{1}$ Department of Radiation Oncology, Medical Center, Faculty of Medicine, University of Freiburg, Robert-Koch-Str. 3, 79106 Freiburg, Germany Full list of author information is available at the end of the article
}

C C The Author(s). 2020 Open Access This article is licensed under a Creative Commons Attribution 4.0 International License, which permits use, sharing, adaptation, distribution and reproduction in any medium or format, as long as you give appropriate credit to the original author(s) and the source, provide a link to the Creative Commons licence, and indicate if changes were made. The images or other third party material in this article are included in the article's Creative Commons licence, unless indicated otherwise in a credit line to the material. If material is not included in the article's Creative Commons licence and your intended use is not permitted by statutory regulation or exceeds the permitted use, you will need to obtain permission directly from the copyright holder. To view a copy of this licence, visit http://creativecommons.org/licenses/by/4.0/ The Creative Commons Public Domain Dedication waiver (http://creativecommons.org/publicdomain/zero/1.0/) applies to the data made available in this article, unless otherwise stated in a credit line to the data. 
(Continued from previous page)

Conclusions: RT of the panpharynx and cervical lymph nodes with concurrent chemotherapy in case of risk factors demonstrated good locoregional control, but the metachronous occurrence of distant metastases limited survival and must be further addressed.

Keywords: Head-and-neck cancer, Carcinoma of unknown primary, Lymph node, CUP, Radiotherapy, Chemotherapy

\section{Background}

Cancer of unknown primary is the seventh most common malignant disease in the Western world and constitutes the fourth most common cause for cancer deaths [1]. Although relatively uncommon, management of cervical lymph node metastases from a cancer of unknown primary (CCUP) remains a therapeutic challenge [2]. This condition most often affects older men with nicotine and/or alcohol abuse, and the most common histology remains squamous cell carcinoma (SCC) [3]. Usually, cervical swelling is the first symptom noted by patients, and pain and dysphagia have also been reported to result in the diagnosis of CCUP [4].

In the last decade, national and international recommendations on standardized procedures for CCUP have been updated [5]. Moreover, refinement and further development of diagnostic and therapeutic procedures have improved management and hence resulted in improved outcomes [6-9]. Diagnostic workup includes careful clinical examination, cervical nodal ultrasound and panendoscopy, combined with diagnostic tonsillectomy. Computed tomography $(\mathrm{CT})$, magnetic resonance imaging (MRI), and ${ }^{18} \mathrm{~F}$-fluorodeoxyglucose-positron emission tomography-CT (FDG-PET-CT) are also essential to further clarify the extent of the disease and to assess potential primaries. In this context, the advent of FDG-PET-CT has resulted in detection rates of up to $40 \%$ of occult primaries not amenable to conventional diagnostic imaging and also helps to further clarify the extent of the disease $[10,11]$. In this regard, FDG-PET-CT has been demonstrated as a cost-effective measure in patients with N1 and N2 status [12].

Today, molecular analyses and assessment of human papillomavirus (HPV) and Epstein-Barr virus (EBV) status provide additional diagnostic and prognostic information. As demonstrated for other SCCs of the headand-neck region, HPV positivity influences the prognosis of CCUP patients, and the presence of ECS in p16positive tumors does not seem to affect survival [8]. However, de-escalation approaches for these cases are not recommended outside of clinical trials. The real impact of the available diagnostic means on clinical decision-making and therapeutic approaches remains unclear and illustrates broad differences between individual centers.
In early stage disease $(\mathrm{cN} 1 / \mathrm{pN} 1)$ without additional risk factors, local single-modality treatment (surgery or radiotherapy) constitutes the treatment standard [13], but for advanced disease, the extent of local therapies remain controversial, especially regarding treatment of elective nodal regions. In the case of radiotherapy, several concepts have been proposed that may encompass partial or total mucosal coverage as well as ipsi- or bilateral lymphatics [14-16].

CCUP management remains a multidisciplinary challenge, owing to the lack of prospective randomized studies. The goal of this analysis was to evaluate patterns of management and resulting oncological outcomes of CCUP in a large single-center patient cohort receiving radiotherapy.

\section{Methods \\ Patients}

This analysis included all patients with histologically proven CCUP treated with radiotherapy between 2008 and 2019 at University of Freiburg Medical Center, Germany. Demographic and clinical data were retrospectively taken from electronic patient records. At the time of therapy, no primary tumor had been identified in any patient following the diagnostic workup delineated below. In all patients, therapy was based on recommendations of the multidisciplinary tumor board. Due to the time period included in this analysis, HPV and EBV status was not routinely tested. Pathological data were taken from the pathology reports. Tumor classification was determined on the basis of pathological reports and contrast-enhanced imaging and was encoded according to the 7th edition of the UICC TNM classification. "Smokers" referred to a smoking history of at least 10 years. Ethical approval was obtained from Freiburg University Independent Ethics Committee for this analysis (record no. 555/18).

\section{Diagnostic procedures and surgery}

Primary workup included a detailed clinical examination, contrast-enhanced CT of the neck, thorax and abdomen, and ultrasound of the neck. FDG-PET-CT and MRI of the neck or abdomen were performed at clinical discretion based on availability and time period. 
Panendoscopy with esophagoscopy was conducted, and, if not yet performed, a diagnostic tonsillectomy was completed. For limited stages (cN1) without neck dissection due to patient comorbidities or patient wishes, fine needle aspiration (FNA) or selective extirpation of affected lymph nodes were performed. Up-front neck dissection (ND) of the affected sides was completed for all other patients.

\section{Radiation treatment and chemotherapy}

Patients received image-guided radiotherapy (IGRT), mainly using intensity-modulated techniques (IMRT) (Fig. 1). All patients were immobilized with a head-neck thermoplastic mask and underwent a planning CT scan. A margin of $0.5-1 \mathrm{~cm}$ was added to the gross tumor volume (GTV) for clinical target volume (CTV) delineation in order to treat microscopic spread, and a PTV margin of $0.3-0.5 \mathrm{~cm}$ was added for including organ motion and set up-errors. Radiotherapy planning was performed using the Oncentra MasterPlan ${ }^{\circ}$ (Nucletron BV, Veenendaal, the Netherlands) and Eclipse ${ }^{\mathrm{Tn}}$ planning systems (Varian Medical Systems). Elective clinical target volumes (CTV) included lymph node levels $\mathrm{Ib}$ to $\mathrm{V}$ on the affected cervical sides and level II to $\mathrm{V}$ for unaffected sides as well as oropharyngeal, hypopharyngeal and laryngeal mucosa. Oral cavity or nasopharyngeal mucosa
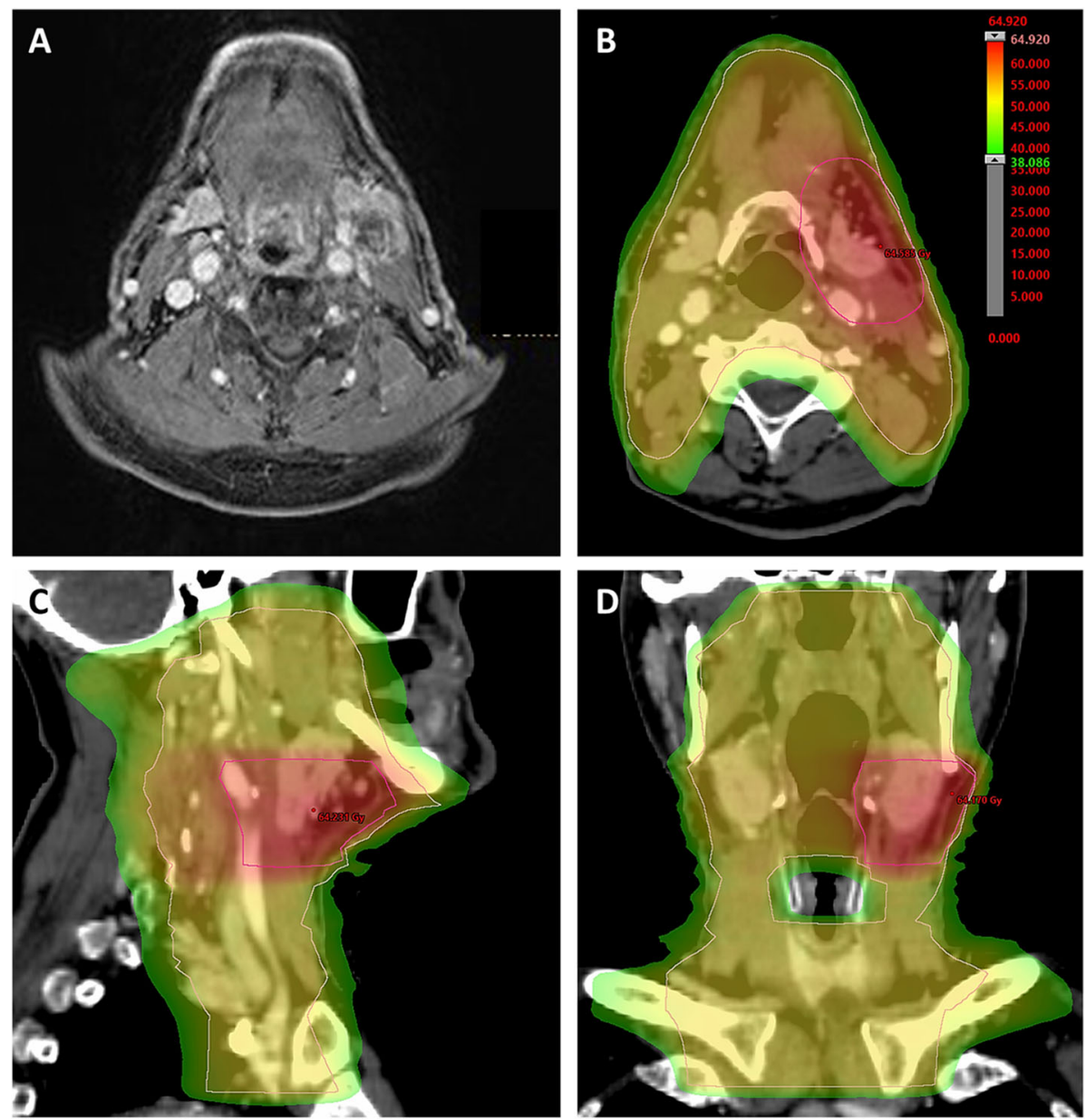

Fig. 1 Adjuvant chemoradiotherapy for a CCUP in a 70-year-old male patient. (A) Pretherapeutic sonography, CT and MRI imaging (a) in December 2017 showed a pathological lymph node in level lla on the left side and a suspicious lymph node in level II on the right side. A panendoscopy with multiple biopsies of different mucosal regions revealed no primary tumor. As the patient had a tonsillectomy as child, no additional tonsillectomy was performed. The recommended FDG-PET-CT was not conducted, as the costs were not covered by the patient's health insurance. After bilateral ND in January 2018, pathological assessments showed one necrotic lymph node $(1.4 \mathrm{~cm}$ diameter) with poorly differentiated (G3), HPV-positive squamous cell carcinoma cells in left-sided level lla, giving a cTx pN1 cMO CCUP according to the 7th Edition of the UICC TNM classification. Based on the recommendations of the multidisciplinary tumor board, an adjuvant cisplatin-based chemoradiotherapy with intensity-modulated radiotherapy was performed between March and April 2018. The elective lymphatic drainage and mucosa received 50 Gy in 25 fractions, while the high-risk PTV was treated with a sequential boost of 10 Gy delivered in 5 fractions. (b, c and d) Dose distribution of a volumetric modulated arc therapy plan in an axial (b), sagittal (c) and coronary (d) scan image. The last follow-up in March 2019 showed no signs of recurrence 
was included depending on the location of the lymph node metastases.

The elective lymphatic drainage and mucosa was treated to a total dose of 50-54 Gy in five weekly fractions of 1.8-2.0 Gy. Macroscopic lymph nodes (if not resected by ND) received 66-70 Gy using a simultaneous or sequential boost concept. After resection, the surgical lymph node bed was treated to a total dose of 60-66 Gy depending on pathologic findings and the presence of ECS. Risk structures were outlined on the planning CT and protected in accordance with QUANTEC recommendations [17].

\section{Outcome measures}

Follow-up care included clinical examinations including endoscopy and radiological imaging with ultrasound as well as CT and/or MRI. Follow-up examinations including imaging were performed every 3 months for the first 3 years and every 6 months between years 3 and 5 . Patients, who were lost to follow-up, were censored for statistical analyses. Local or locoregional relapse, distant progression and overall survival (OS) were analyzed for each patient. OS was defined as the period from the start of therapy to the last contact or death. Based on followup radiological imaging, locoregional progression-free survival (PFS) was assessed and defined as the time from treatment initiation to locoregionally progressive disease or death. Distant metastasis-free survival (DMFS) was defined as newly diagnosed distant metastases on followup radiological imaging or death.

\section{Statistical analysis}

Statistical analysis was performed with R Studio version 3.6.1. The Kaplan-Meier estimator using log-rank test was applied for OS as well as for PFS and DMFS. Additionally, univariate Cox-regression analyses were performed to assess the effect of several clinical factors on OS (smoking, advanced nodal status, age, gender, ECS, tumor grading, ND and utilization of FDG-PET-CT). A $p$-value of $<0.05$ was considered to be statistically significant. Since this was an exploratory trial, all $p$-values were interpreted descriptively and no adjustment for multiple testing was applied.

\section{Results}

\section{Patient and treatment characteristics}

Fifty-eight patients receiving IGRT and mainly IMRT for CCUP were included in this analysis. The median age of the analyzed patient cohort was 62 years (range 37-92), and patients were predominantly male $(n=44,75.9 \%)$. The majority of patients presented with pN2-pN3 disease (36 patients, $80.0 \%$ ). ECS was present in 21 patients (36.2\%), and 37 patients (63.8\%) had high-grade disease. The majority of affected lymph nodes were located in level II $(n=35)$ or level III $(n=10)$, and the most prevalent histology was squamous cell carcinoma $(n=45$; 77.6\%). While 2 patients (3.4\%) exhibited EBV-positive lymph nodes, 7 tumors (12.1\%) were tested positive for HPV. Patient characteristics are summarized in Table 1.

Nearly all patients received an ultrasound examination (Table 2). CT of the neck and thorax was performed in 45 patients (77.6\%), whereas FDG-PET-CT was performed in 30 patients (51.7\%). Fifty-two patients were investigated by panendoscopy $(89.7 \%)$. Treatment included unilateral ND in 23 patients (39.7\%) and bilateral ND in 15 patients (25.9\%). ND was omitted in $22.4 \%$ of patients. Simultaneously or sequentially to panendoscopy/ND, a unilateral or bilateral tonsillectomy was performed in 37 patients $(63.8 \%)$.

\section{Radiotherapy and chemotherapy characteristics}

The majority of patients received adjuvant radiotherapy after lymph node dissection $(n=35 ; 60.3 \%) ; 21$ patients (36.2\%) were treated with definitive radiotherapy, and 2 patients $(3.4 \%)$ were scheduled for palliative radiotherapy, performed in the setting of oligometastatic disease (Table 3). Radiotherapy was completed in 52 patients (89.7\%), with the primary reason for therapy discontinuation being deterioration of the general condition and/or progressive disease. A sequential or simultaneous integrated boost to the macroscopic tumor or tumor bed was applied in 48 patients (82.8\%).

Concurrent chemotherapy was prescribed in 40 patients (69.0\%); indications for simultaneous chemotherapy were ECS and residual tumor. In 6 patients, concurrent chemotherapy was recommended by the multidisciplinary tumor board for adenocarcinoma or undifferentiated histologies and rapid tumor growth. Twenty-seven patients (67.5\%) received cisplatin monotherapy $\left(100 \mathrm{mg} / \mathrm{m}^{2}\right.$ body surface area every 3 weeks) (supplementary table 1 , supplementary table 2). Carboplatin monotherapy was administered in 5 patients (12.5\%), and lower-dose cisplatin combined with 5fluorouracil in 4 patients (10.0\%). Cetuximab was applied in 1 case on an individual basis. Twenty-six patients (65.0\%) completed the planned chemotherapy cycles. Induction chemotherapy was not performed in any patient.

\section{Patient outcomes}

Median follow-up in this patient cohort was 29.9 months (range 4.6-121.9 months). Median OS was not reached, and 1-year OS, 3-year OS and 5-year OS were 81,2, $64.9 \%$ and $56,6 \%$, respectively (Fig. 2 a). Restricting the analysis to CCUP patients with SCC, 1-year OS, 3-year OS and 5-year OS ranged at $83.3,68.6$ and $62.4 \%$, respectively. There was no significant OS difference between CCUP patients with SCC and non-SCC histology (supplementary Figure 1) $(p=0.48$, log-rank test). Seven patients (12.1\%) developed in-field lymph node 
Table 1 Patient characteristics regarding CCUP patients treated by radiotherapy in our institution between 2008 and 2019 ( $n=$ 58). If both a clinical nodal status ( $\mathrm{cN}$ ) and a pathological nodal (pN) status were available, only pN was stated

\begin{tabular}{lll}
\hline Variable & & \\
\hline Age (median, range) & $62(37-92)$ & \\
\hline Sex & $\mathbf{n}$ & $\%$ \\
female & 14 & 24.1 \\
male & 44 & 75.9
\end{tabular}

\section{ECOG}

0

1

unknown

Smoking

no

yes

unknown

Localization

।

॥

III

IV

VIII

unknown

$\mathrm{cN}, n=13$

CN1

$\mathrm{CN} 2$

$\mathrm{cN} 3$

unknown

$\mathrm{pN}, \mathrm{n}=45$

$\mathrm{pN} 1$
$\mathrm{pN} 2$
$\mathrm{pN} 3$
0
1
$\mathrm{x}$
unknown

\section{Histology}

squamous cell carcinoma

adenocarcinoma

undifferentiated

others'

unknown

\section{Grading}

1
50.0

20.7

3.4

65.5

17.2

3.4

6.9

3.4

7.7

61.5

23.1
Table 1 Patient characteristics regarding CCUP patients treated by radiotherapy in our institution between 2008 and 2019 ( $n=$ 58). If both a clinical nodal status ( $\mathrm{CN}$ ) and a pathological nodal (pN) status were available, only pN was stated (Continued)

\begin{tabular}{lll}
\hline Variable & & \\
\hline Age (median, range) & 62 (37-92) & \\
\hline 3 & 37 & 63.8 \\
unknown & 7 & 12.1 \\
HPV/EBV & & \\
HPV-positive & 7 & 12.1 \\
EBV-positive & 2 & 3.4 \\
ECS & & \\
no & 27 & 46.6 \\
yes & 21 & 36.2 \\
unknown & 10 & 17.2 \\
\hline sarcomatoid carcinoma, lymphoepithelial carcinoma
\end{tabular}

relapse during the follow-up period, and median locoregional PFS was 31 months, with 1-year PFS, 3-year PFS and 5-year PFS amounting to 70.0, 49.3 and $49.3 \%$, respectively (Fig. 2b). Locoregional PFS exclusively for SCC CCUP was 73.8, 57.9 and $57.9 \%$ after 1 year, 3 years and 5 years, respectively. Distant metastases were diagnosed in 13 patients $(22.4 \%)$ after treatment. Median DMFS was 27.5 months with 1-year DMFS, 3-year DMFS and 5-year DMFS ranging at 67.1, 48.9 and $45.4 \%$, respectively (Fig. 2c). If limited to SCC as CCUP histology, 1-year DMFS, 3-year DMFS and 5-year DMFS amounted to $71.1,56.7$ and $52.6 \%$, respectively.

Univariate analyses demonstrated that smoking was significantly associated with impaired OS (HR $=5.34,95 \% \mathrm{CI}$ 1.21-23.55, $p<0.05$ ) (Fig. 3a, Table 4). There was a trend towards reduced OS for CCUP patients with $\mathrm{pN} 3$ (pN3 versus $\mathrm{pN} 1$ : $\mathrm{HR}=3.76,95 \% \mathrm{CI} 0.91-15.55, p=0.07$ ), and log-rank tests demonstrated significantly reduced OS for advanced nodal status $(p<0.05$, log-rank tests) (Figs. 3b). Cox regression analyses of age, gender, tumor histology, tumor grading, ECS, utilization of FDP-PET-CT, ND and concomitant chemotherapy did not demonstrate an effect on survival ( $p>0.05$ for all) (Fig. 3c and d).

\section{Acute toxicities}

Acute toxicities were assessed during radiotherapy as well as the first 90 days after completion of treatment and were quantified using National Cancer Institute-Common Terminology Criteria for Adverse Events (CTCAE v4.03). Overall, rates of grade 3 treatment-related acute toxicities were moderate and were observed for radiation dermatitis in 3 patients $(5.2 \%)$ and for oral mucositis in 12 patients (20.7\%) (Table 5). Only 1 patient (1.7\%) developed grade 4 mucositis, and there were no grade 5 events. Chronic treatment-related toxicities could not be systematically 
Table 2 Diagnostic work-up for CCUP patients treated in our institution between 2008 and 2019

\begin{tabular}{|c|c|c|}
\hline Diagnostics & $n$ & $\%$ \\
\hline \multicolumn{3}{|l|}{ Sonography neck } \\
\hline no & 2 & 3.4 \\
\hline yes & 53 & 91.4 \\
\hline unknown & 3 & 5.2 \\
\hline \multicolumn{3}{|l|}{ CT } \\
\hline no & 6 & 10.3 \\
\hline head & 3 & 5.2 \\
\hline head/thorax & 45 & 77.6 \\
\hline head/thorax/abdomen & 4 & 6.9 \\
\hline \multicolumn{3}{|l|}{ Panendoscopy } \\
\hline no & 6 & 10.3 \\
\hline yes & 52 & 89.7 \\
\hline \multicolumn{3}{|l|}{ GI endoscopy } \\
\hline no & 15 & 25.9 \\
\hline yes & 42 & 72.4 \\
\hline unknown & 1 & 1.7 \\
\hline \multicolumn{3}{|l|}{ MRI neck } \\
\hline no & 32 & 55.2 \\
\hline yes & 25 & 43.1 \\
\hline unknown & 1 & 1.7 \\
\hline \multicolumn{3}{|l|}{ PET-CT } \\
\hline no & 28 & 48.3 \\
\hline yes & 30 & 51.7 \\
\hline \multicolumn{3}{|l|}{ Neck dissection } \\
\hline no & 13 & 22.4 \\
\hline neck dissection unilateral & 23 & 39.7 \\
\hline neck dissection bilateral & 15 & 25.9 \\
\hline others $^{1}$ & 7 & 12.1 \\
\hline \multicolumn{3}{|l|}{ Tonsillectomy } \\
\hline vno & 17 & 29.3 \\
\hline yes & 37 & 63.8 \\
\hline unknown & 4 & 6.9 \\
\hline
\end{tabular}

'sampling, lymph node extirpation

assessed due to the lack of systematic long-term follow-up information.

\section{Discussion}

Our data derived from a large cohort of patients receiving IGRT and mostly IMRT for CCUP demonstrate that radiotherapy as part of a multidisciplinary treatment approach including is an effective treatment modality for these patients and results in relatively high locoregional control rates with moderate higher-grade toxicities. Nevertheless, the data show that systemic control remains a challenge in these patients as reflected in median DMFS rates of only
Table 3 Treatment characteristics in our CCUP patient cohort

\begin{tabular}{|c|c|c|}
\hline Treatment & $\mathrm{n} /$ median & $\%$ \\
\hline \multicolumn{3}{|l|}{ Radiotherapy } \\
\hline definitive & 21 & 36.2 \\
\hline adjuvant & 35 & 60.3 \\
\hline palliative & 2 & 3.4 \\
\hline \multicolumn{3}{|l|}{ Completion radiotherapy } \\
\hline no & 4 & 6.9 \\
\hline yes & 52 & 89.7 \\
\hline unknown & 2 & 3.4 \\
\hline \multicolumn{3}{|c|}{ Radiotherapy dose (median, range) } \\
\hline Total dose (including boost) & $60.0 \mathrm{~Gy}(18.0-72.0)$ & \\
\hline \multicolumn{3}{|l|}{ Single radiation dose } \\
\hline $1.7 \mathrm{~Gy}$ & 1 & 1.7 \\
\hline $1.8 \mathrm{~Gy}$ & 3 & 5.2 \\
\hline $2 \mathrm{~Gy}$ & 52 & 89.7 \\
\hline $3 \mathrm{~Gy}$ & 2 & 3.4 \\
\hline \multicolumn{3}{|l|}{ Boost, $n=48$} \\
\hline integrated & 4 & 8.3 \\
\hline sequential & 44 & 91.7 \\
\hline \multicolumn{3}{|l|}{ Simultaneous chemotherapy } \\
\hline no & 18 & 31.0 \\
\hline yes & 40 & 69.0 \\
\hline \multicolumn{3}{|l|}{ Chemotherapy, $n=40$} \\
\hline cisplatin & 27 & 67.5 \\
\hline cisplatin/5-fluorouracil & 4 & 10.0 \\
\hline cetuximab & 1 & 2.5 \\
\hline carboplatin & 5 & 12.5 \\
\hline others ${ }^{1}$ & 3 & 5.0 \\
\hline \multicolumn{3}{|c|}{ Completion chemotherapy, $n=40$} \\
\hline no & 6 & 15.0 \\
\hline yes & 26 & 65.0 \\
\hline unknown & 8 & 20.0 \\
\hline
\end{tabular}

${ }^{1}$ mitomycin C/5-fluorouracil, cisplatin/vinorelbine,

cisplatin/mitomycin C/5-fluorouracil

27.5 months. With a median age of 62 years, predominantly male patients and SCC as the most common histology, our patient cohort is comparable to previous CCUP studies in terms of demographic parameters [2,18-21]. The majority of lymph node metastases were observed in the upper neck with level II as the most frequent localization, suggesting an occult head-and-neck cancer in the majority of patients [5].

Compared to other retrospective analyses, our oncological results in terms of OS and locoregional control are quite favorable [18]. While older series focused on conventional 2or 3-dimensional radiotherapy techniques reported 5-year survival rates between 22 and 53\%, our cohort exhibited a 5 -year OS of almost $60 \%$ [4, 22-25]. Our results especially 

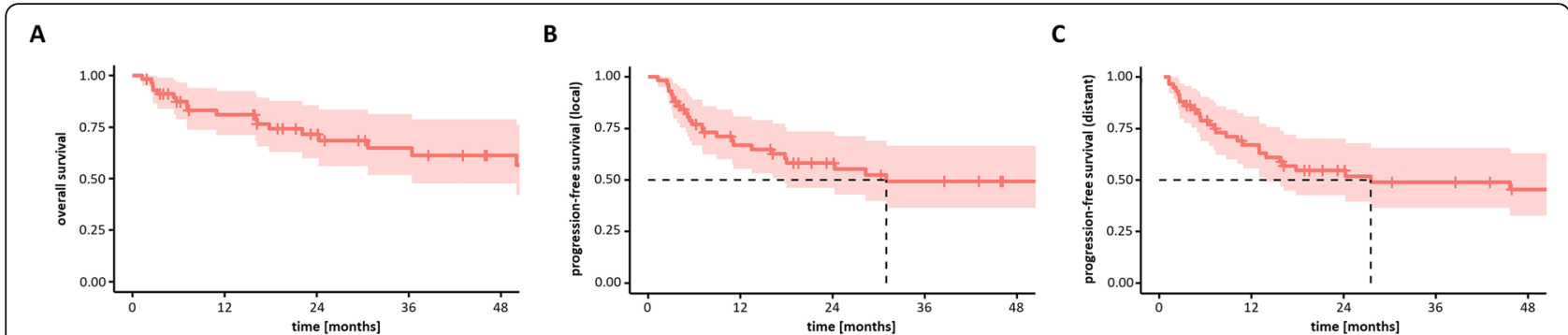

Fig. 2 Kaplan-Meier curves showing OS (a), PFS (locoregional) (b) and DMFS (c) of CCUP patients treated by radiotherapy $(n=58)$. The red area shows the $95 \%$ confidence intervals for the survival rates

hold up favorably as we did not limit our analysis to squamous cell CCUP, which commonly has a favorable prognosis compared to other cervical histologies $[13,26]$. Other studies including only SCC CCUP reported about 5-year OS rates ranging at 40\% [19], 47\% [27], 48\% [21] and 52\% [28], which is lower than the 5-year OS of $62 \%$ in SCC CCUP patients in our analysis.

Diagnostic tonsillectomy was performed in $63.8 \%$ of our CCUP patients. It has been previously demonstrated that tonsillectomy resulted in the diagnosis of primary tonsillar carcinoma in about one quarter of patients that initially presented with CCUP [29]. Additionally, previous data suggest that a diagnostic workup supplementing imaging and panendoscopy with a bilateral tonsillectomy was found to lead to tumor detection rates of $59.6 \%$, which were superior to imaging alone, and survival rates for CCUP were found improved if a tonsillectomy was performed $[4,30]$.

The relevance of individual diagnostic procedures has remained somewhat controversial, but the widespread utilization of FDG-PET-CT in the last years has increased detection rates of primary cancer sites in CCUP. Several analyses quantifying the use of FDG-PET-CT for CCUP
A

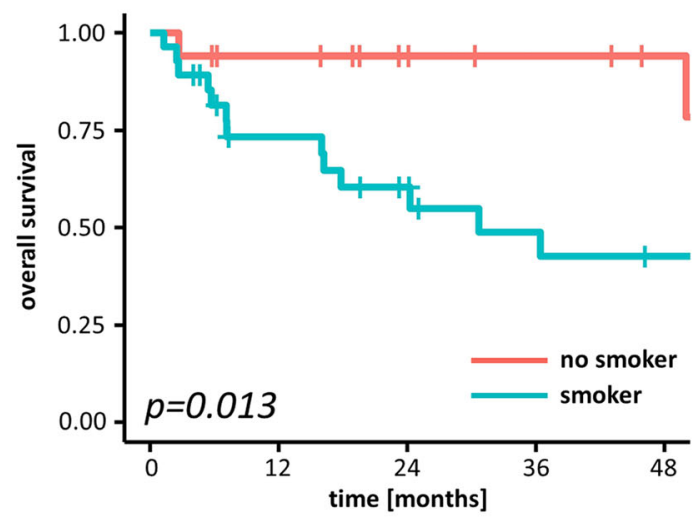

B

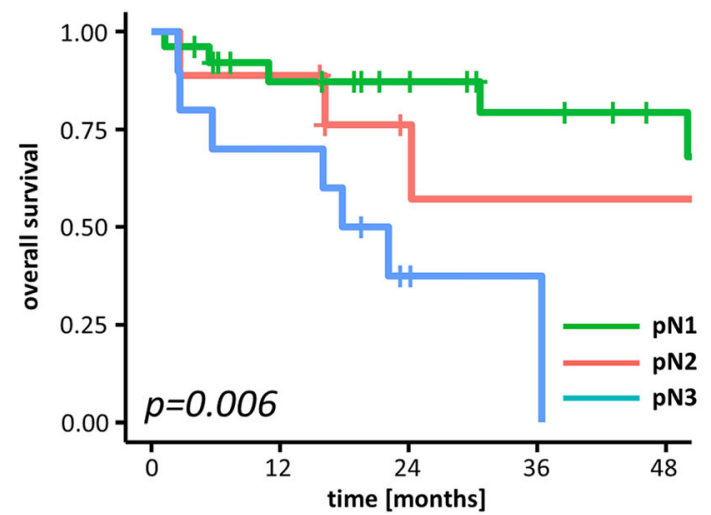

C

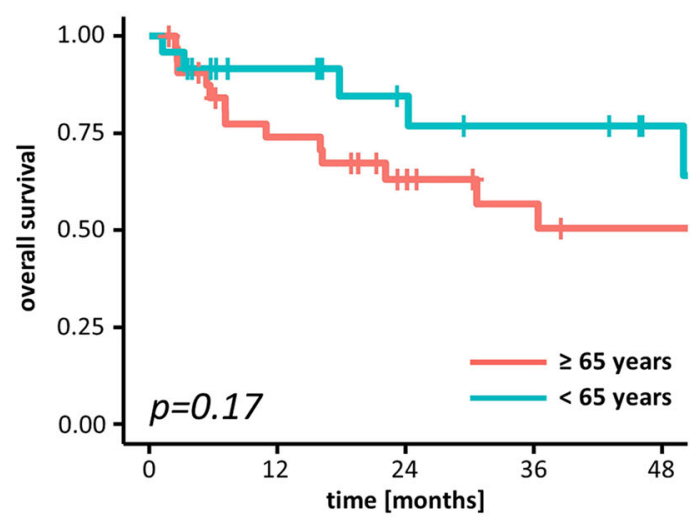

D

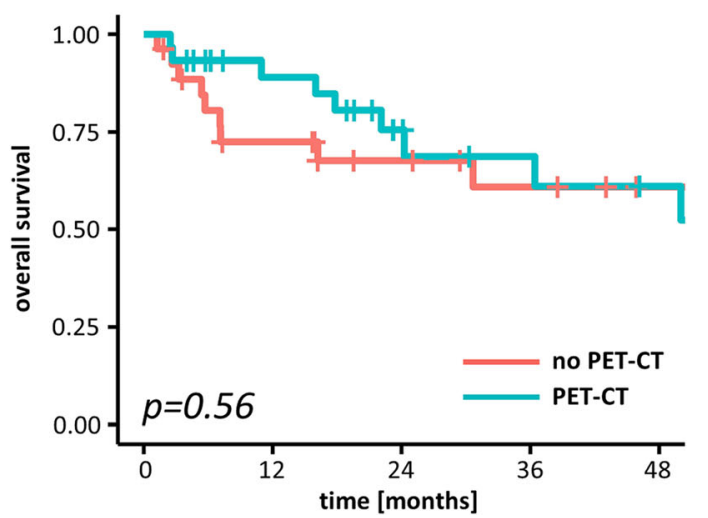

Fig. 3 Kaplan-Meier curves demonstrating OS according to smoking status (a), pathological nodal status (b), age (c) and FDG-PET-CT imaging (d). Log-rank tests were performed to compare the groups 
Table 4 Cox-regression for clinical and pathological parameters regarding OS effects

\begin{tabular}{llll}
\hline Parameter & HR & Cl 95\% & $\boldsymbol{p}$-value \\
\hline Age $\geq 65$ / < 65 years & 2.04 & $0.72-5.56$ & 0.18 \\
Sex male / female & 1.25 & $0.36-4.36$ & 0.73 \\
Smoker / non-smoker & 5.34 & $1.21-23.55$ & 0.03 \\
No SCC / SCC & 1.72 & $0.38-7.69$ & 0.49 \\
Grading G3 / G2 & 1.22 & $0.34-4.30$ & 0.76 \\
pN3 / pN1 & 3.76 & $0.91-15.55$ & 0.07 \\
pN2 / pN1 & 0.67 & $0.17-2.70$ & 0.57 \\
pN2b-pN3 / pN1-pN2a & 1.49 & $0.54-4.17$ & 0.43 \\
ECS / no ECS & 1.73 & $0.68-4.39$ & 0.25 \\
No PET-CT / PET-CT & 1.30 & $0.53-3.23$ & 0.56 \\
No ND / unilateral ND & 1.52 & $0.52-4.45$ & 0.45 \\
Bilateral ND / unilateral ND & 0.91 & $0.30-2.78$ & 0.87 \\
No ND / any ND & 1.79 & $0.67-4.76$ & 0.24 \\
No Chemotherapy / chemotherapy & 0.63 & $1.92-2.07$ & 0.42 \\
\hline
\end{tabular}

have reported overall primary tumor detection rates ranging between 24.5 and 37\% [10, 11]. In our analysis, FDGPET-CT utilization was not associated with improved OS. On the one hand, this could be due to the lack of power of the hypothesis test, as our sample size is quite small. On the other hand, considering that the majority of CCUP cases are due to underlying head-and-neck cancers, the lack of an effect for FDG-PET-CT imaging could also be due to the extensive coverage of the head-and-neck region including the complete bilateral lymphatics and mucosa in our patient cohort. However, the widespread utilization of PET imaging for CCUP staging may help to de-escalate the treatment volumes to the involved sites or laterality in order to reduce treatment-related toxicities [31]. Furthermore, as utilization of FDG-PET-CT in the diagnostic work-up may have facilitated detection of the primary tumor, some patients with initial suspicion of CCUP may have received de-intensified treatments after detection of the primary tumor. Based on the results from the prospective DAHANCA-13 trial, PET-CT imaging is now recommended as part of the diagnostic workup for CCUP patients [6].

Another controversial topic pertains to the extent of radiotherapy, especially the need for uni- versus bilateral treatment to the elective neck. A meta-analysis failed to demonstrate a survival benefit for elective bilateral neck radiotherapy in comparison to unilateral treatment [32]. However, in a large retrospective Danish study, the 5-year control rates were reported to be $51 \%$ for bilateral radiotherapy versus only $27 \%$ for ipsilateral radiotherapy accompanied by a trend towards improved disease-specific survival; however, the staging means were not comparable to the current standards [2]. The EORTC-24001-22005
Table 5 Toxicity results of several (chemo)radiotherapy-related acute side effects according to the Common Terminology Criteria for Adverse Events (CTCAE) v5.0

\begin{tabular}{cll}
\hline Toxicity & $\mathrm{n}$ & $\%$ \\
\hline Dermatitis & 9 & 15.5 \\
0 & 25 & 43.1 \\
1 & 21 & 36.2 \\
2 & 3 & 5.2 \\
3 & & \\
Dysphagia & 8 & 13.8 \\
0 & 15 & 25.9 \\
1 & 34 & 58.6 \\
2 & 1 & 1.7 \\
3 & & \\
Nausea & 33 & 56.9 \\
0 & 22 & 37.9 \\
1 & 3 & 5.2 \\
2 &
\end{tabular}

Mucositis

0

2

3

4

Xerostomia

0

1

2

Hoarseness

0

1

Dyspnea

0

Dysgeusia

0

15.5

1

2

55.2

Pain

$\begin{array}{lll}0 & 21 & 36.2\end{array}$

$\begin{array}{lll}1 & 25 & 43.1\end{array}$

2

12

trial aimed to clarify this issue by conducting a prospective randomized trial comparing ipsilateral versus bilateral neck radiotherapy for CCUP patients but was unable to provide results owing to low patient enrollment. A recent meta- 
analysis reported an improvement regarding locoregional tumor control rates, but not disease-free survival for bilateral radiotherapy compared to ipsilateral radiotherapy [33]. The majority of the published studies did not provide routine FDG-PET-CT-based staging of the neck, and considering the lower sensitivity of other diagnostic imaging modalities, contralateral lymph node metastases may have been missed in a subgroup of patients. Bilateral radiotherapy, especially when combined with radiotherapy to large mucosal areas was reported to provoke high toxicity rates for CCUP patients, but these patients were treated with older 2- or 3-dimensional techniques [33]. Our study focused on patients that were treated with IGRT and IMRT, as these newer techniques have demonstrated superior toxicity profiles for head-and-neck treatments in various analyses [2, 22, 23, 34-36]. Hence, in our cohort, only $5.2 \%$ of patients exhibited acute grade 3 radiation dermatitis and $22.4 \%$ of patients had acute grade $\geq 3$ mucositis with no reported grade 5 toxicities, demonstrating an acceptable tolerance for extended radiotherapy. The moderate acute toxicity rates were mirrored in the high radiotherapy completion rates in about $90 \%$ of patients. It should be noted that the mucosal doses varied between 50 and 54 Gy in our cohort, which is lower than in most other series [21, 37, 38]. The current NCCN guideline recommends irradiation doses to the putative mucosal sides ranging at 50-60 Gy in combination with concomitant systemic treatment; if radiotherapy is administered alone, 50-66 Gy are proposed. Therefore, the lower mucosal radiation dose may contribute to the favorable toxicity profile in our study cohort.

In our cohort, positive smoking history was found to significantly impair survival of CCUP patients treated by radiotherapy. Tribius and colleagues reported about an association between smoking and HPV-status in CCUP patients with significantly more HPV-positive tumors in smokers [37]. Furthermore, tobacco history negatively affected survival of patients with HPV-positive CCUPs in this study. As HPV-testing was not routinely performed in the time span of our study, we were not able to investigate the relationship between smoking history and HPV-status in CCUP patients. However, smoking should be considered as a risk factor for CCUP patients, especially in the case of HPV-positive tumors. In a study performed by Dixon and coworkers, CCUP patients with p16-positive tumors exhibited less advanced nodal status and superior disease-free survival [39]. The observed trend towards improved survival in CCUP patients with N1-status may therefore also partially related to the HPV-status.

Whether radiotherapy can be omitted altogether after primary surgery is another matter of debate. Especially for pN1 or pN2a stages without ECE, omission of radiotherapy may be justified if a close and imaging-based follow-up is guaranteed, so that salvage radiotherapy can be applied in case of progression. However, in a large study comprising data from 5 Danish centers, patients treated with surgery alone exhibited a significantly elevated risk of emerging primary compared to those receiving adjuvant radiotherapy [2]. 5-year risk for emerging primary was $54 \%$ in the surgery group versus $15 \%$ in the adjuvant RT group [2]. Therefore, to date, postoperative radiotherapy is warranted for the large majority of CCUP patients.

The use and benefit of concomitant chemoradiotherapy in CCUP patients has been a matter of debate and largely lacks clinical evidence due to missing prospective studies $[5,40]$. The indications for the utilization of concomitant systemic treatment are extrapolated from data on other head-and-neck cancers, and platinum-based agents are most commonly used for concomitant chemotherapy [40, 41]. In a study by Chen and colleagues, concomitant chemotherapy was found to result in increased toxicity rates but no improvements of OS, PFS or locoregional control rates [40]. Due to the retrospective nature of this study, it should be noted that simultaneous chemotherapy may have been applied especially for CCUPs with highrisk features or more advanced disease, suggesting a potential bias to the disadvantage of concurrent chemotherapy utilization. In our cohort, chemotherapy was most commonly prescribed for macroscopic tumor, ECS or positive resection margins according to the established high-risk features for head-and-neck SCC, but did not result in an improvement of patient survival [42-44].

While our analysis is one of the first comprehensive datasets presenting outcome and toxicities in CCUP patients undergoing IGRT and mainly IMRT, it has several limitations due to its retrospective nature and small sample size. Prospective studies for relatively rare disease constellations are difficult to conduct, and especially prospective studies comparing radiotherapy with surgery exhibit a high risk of failure due to insufficient accrual [45]. However, despite the aforementioned challenges, prospective, multi-center trials will be eventually needed in order to identify the ideal diagnostic and treatment algorithm for CCUP patients. Additionally, regarding the occurrence of distant metastases in almost 1 of 4 CCUP patients, further research will need to focus on effective systemic treatments as part of adjuvant therapy in order to avoid distant relapse.

Taken together, out dataset demonstrated excellent results and moderate toxicity in CCUP patients when treated with extensive radiotherapy based on modern radiation techniques.

\section{Supplementary information}

Supplementary information accompanies this paper at https://doi.org/10. 1186/s13014-020-01529-z.

Additional file 1 Supplementary Figure 1. Kaplan-Meier curves showing OS for non-SCC CCUP and SCC CCUP patients. The $p$-value indicates the result of the log-rank test. 
Additional file 2. Supplementary Table 1: Administration of concomitant chemotherapy depending on the histology of CCUP.

Additional file 3. Supplementary Table 2: Specification of concomitant chemotherapy in dependence of the CCUP histology.

\section{Acknowledgements}

Not applicable.

\section{Authors' contributions}

T.S., N.H.N.: Study conception and study design. T.S., A.R., T.K., R.S.: Data acquisition and data analysis. K.H.: Statistical analysis. T.S., A.R., T.K., V.V., N.H.N.: Data interpretation, manuscript preparation, manuscript editing. T.S., A.R., K.H. V.V., C.Z., J.F., R.L., A.K., A.L.G., N.H.N.: Critical discussion of the manuscript. All authors read and approved the final manuscript.

\section{Author's information}

Alexander Rühle was supported by the IMM-PACT-Programme for Clinician Scientists, Department of Medicine II, Medical Center - University of Freiburg and Faculty of Medicine, University of Freiburg, funded by the Deutsche Forschungsgemeinschaft (DFG, German Research Foundation) - 413,517,907.

\section{Funding}

This research did not receive any specific grant from funding agencies in the public, commercial, or not-for-profit sectors.

\section{Availability of data and materials}

The datasets used and analyzed during the current study are available from the corresponding author on reasonable request.

\section{Ethics approval and consent to participate}

The study was approved by the institutional ethical review committee (record no. 555/18).

\section{Consent for publication}

Not applicable.

\section{Competing interests}

The authors declare that they have no competing interests.

\section{Author details}

'Department of Radiation Oncology, Medical Center, Faculty of Medicine, University of Freiburg, Robert-Koch-Str. 3, 79106 Freiburg, Germany. ${ }^{2}$ German Cancer Consortium (DKTK) Partner Site Freiburg, German Cancer Research Center (dkfz), Heidelberg, Germany. ${ }^{3}$ Department of Statistics, TU Dortmund University, Dortmund, Germany. ${ }^{4}$ Department of Radiation Oncology, Allegheny General Hospital, Pittsburgh, PA, USA. ${ }^{5}$ Department of Otorhinolaryngology, Medical Center, Faculty of Medicine, University of Freiburg, Freiburg, Germany.

\section{Received: 18 February 2020 Accepted: 2 April 2020}

\section{Published online: 15 April 2020}

\section{References}

1. Pavlidis N, Pentheroudakis G. Cancer of unknown primary site. Lancet. 2012; 379(9824):1428-35.

2. Grau C, Johansen LV, Jakobsen J, Geertsen P, Andersen E, Jensen BB. Cervical lymph node metastases from unknown primary tumours. Results from a national survey by the Danish Society for Head and Neck Oncology. Radiother Oncol. 2000;55(2):121-9.

3. Pfeiffer J, Kayser L, Ridder GJ. Minimal-invasive core needle biopsy of head and neck malignancies: clinical evaluation for radiation oncology. Radiother Oncol. 2009:90(2):202-7.

4. Issing WJ, Taleban B, Tauber S. Diagnosis and management of carcinoma of unknown primary in the head and neck. Eur Arch Otorhinolaryngol. 2003: 260(8):436-43.

5. von der Muller Grun J, Tahtali A, Ghanaati S, Rodel C, Balermpas P. Diagnostic and treatment modalities for patients with cervical lymph node metastases of unknown primary site - current status and challenges. Radiat Oncol. 2017;12(1):82.
6. Johansen J, Buus S, Loft A, Keiding S, Overgaard M, Hansen HS, et al. Prospective study of 18FDG-PET in the detection and management of patients with lymph node metastases to the neck from an unknown primary tumor. Results from the DAHANCA-13 study. Head Neck. 2008;30(4): 471-8.

7. Aslani M, Sultanem K, Voung T, Hier M, Niazi T, Shenouda G. Metastatic carcinoma to the cervical nodes from an unknown head and neck primary site: is there a need for neck dissection? Head Neck. 2007;29(6):585-90.

8. Kharytaniuk N, Molony P, Boyle S, O'Leary G, Werner R, Heffron C, et al. Association of Extracapsular Spread with Survival According to human papillomavirus status in oropharynx squamous cell carcinoma and carcinoma of unknown primary site. JAMA Otolaryngol Head Neck Surg. 2016;142(7):683-90.

9. Lu H, Yao M, Tan H. Unknown primary head and neck cancer treated with intensity-modulated radiation therapy: to what extent the volume should be irradiated. Oral Oncol. 2009;45(6):474-9.

10. Rusthoven KE, Koshy M, Paulino AC. The role of fluorodeoxyglucose positron emission tomography in cervical lymph node metastases from an unknown primary tumor. Cancer. 2004;101(11):2641-9.

11. Kwee TC, Kwee RM. Combined FDG-PET/CT for the detection of unknown primary tumors: systematic review and meta-analysis. Eur Radiol. 2009;19(3): 731-44.

12. Smith KA, Dort JC, Hall SF, Rudmik L. Cost-effectiveness of positron emission tomography-CT in the evaluation of cancer of unknown primary of the head and neck. Head Neck. 2015;37(12):1781-7.

13. Strojan P, Ferlito A, Langendijk JA, Corry J, Woolgar JA, Rinaldo A, et al. Contemporary management of lymph node metastases from an unknown primary to the neck: II. A review of therapeutic options. Head Neck. 2013; 35(2):286-93.

14. Perkins SM, Spencer CR, Chernock RD, Haughey BH, Nussenbaum B, Adkins $\mathrm{DR}$, et al. Radiotherapeutic management of cervical lymph node metastases from an unknown primary site. Arch Otolaryngol Head Neck Surg. 2012; 138(7):656-61.

15. Le NS, Janik S, Simmel H, Erovic BM. Bilateral vs ipsilateral adjuvant radiotherapy in patients with cancer of unknown primary of the head and neck: an analysis of the clinical outcome and radiation-induced side effects. Head Neck. 2019;41(6):1785-94.

16. Richards TM, Bhide SA, Miah AB, Del Rosario $L$, Bodla $S$, Thway $K$, et al. Total mucosal irradiation with intensity-modulated radiotherapy in patients with head and neck carcinoma of unknown primary: a pooled analysis of two prospective studies. Clin Oncol (R Coll Radiol). 2016;28(9):e77-84.

17. Lee TF, Fang FM. Quantitative analysis of normal tissue effects in the clinic (QUANTEC) guideline validation using quality of life questionnaire datasets for parotid gland constraints to avoid causing xerostomia during head-andneck radiotherapy. Radiother Oncol. 2013;106(3):352-8.

18. Al Kadah B, Papaspyrou G, Linxweiler M, Schick B, Rube C, Buchler BS, et al. Cancer of unknown primary (CUP) of the head and neck: retrospective analysis of 81 patients. Eur Arch Otorhinolaryngol. 2017;274(6):2557-66.

19. Christiansen $H$, Hermann RM, Martin A, Nitsche $M$, Schmidberger $H$, Pradier O. Neck lymph node metastases from an unknown primary tumor retrospective study and review of literature. Strahlenther Onkol. 2005;181(6): 355-62.

20. Hauswald H, Lindel K, Rochet N, Debus J, Harms W. Surgery with complete resection improves survival in radiooncologically treated patients with cervical lymph node metastases from cancer of unknown primary. Strahlenther Onkol. 2008;184(3):150-6.

21. Fakhrian K, Thamm R, Knapp S, Molls M, Pigorsch S, Haller B, et al. Radio (chemo) therapy in the management of squamous cell carcinoma of cervical lymph nodes from an unknown primary site. A retrospective analysis. Strahlenther Onkol. 2012;188(1):56-61.

22. Weir L, Keane T, Cummings B, Goodman P, O'Sullivan B, Payne D, et al. Radiation treatment of cervical lymph node metastases from an unknown primary: an analysis of outcome by treatment volume and other prognostic factors. Radiother Oncol. 1995;35(3):206-11.

23. Reddy SP, Marks JE. Metastatic carcinoma in the cervical lymph nodes from an unknown primary site: results of bilateral neck plus mucosal irradiation vs. ipsilateral neck irradiation. Int J Radiat Oncol Biol Phys. 1997;37(4):797-802.

24. Fernandez JA, Suarez C, Martinez JA, Llorente JL, Rodrigo JP, Alvarez JC. Metastatic squamous cell carcinoma in cervical lymph nodes from an unknown primary tumour: prognostic factors. Clin Otolaryngol Allied Sci. 1998:23(2):158-63. 
25. Iganej S, Kagan R, Anderson P, Rao A, Tome M, Wang R, et al. Metastatic squamous cell carcinoma of the neck from an unknown primary: management options and patterns of relapse. Head Neck. 2002;24(3):236-46.

26. Pavlidis N, Briasoulis E, Hainsworth J, Greco FA. Diagnostic and therapeutic management of cancer of an unknown primary. Eur J Cancer. 2003;39(14): 1990-2005.

27. Beldì D, Jereczek-Fossa BA, D'Onofrio A, Gambaro G, Fiore MR, Pia F, et al. Role of Radiotherapy in the Treatment of Cervical Lymph Node Metastases From an Unknown Primary Site: Retrospective Analysis of 113 Patients. Int J Radiation Oncol Biol Phys. 2007;69(4):1051-8.

28. Strojan P, Anicin A. Combined surgery and postoperative radiotherapy for cervical lymph node metastases from an unknown primary tumour. Radiother Oncol. 1998;49(1):33-40.

29. Lapeyre M, Malissard L, Peiffert D, Hoffstetter S, Toussaint B, Renier S, et al. Cervical lymph node metastasis from an unknown primary: is a tonsillectomy necessary? Int J Radiat Oncol Biol Phys. 1997;39(2):291-6.

30. Waltonen JD, Ozer E, Hall NC, Schuller DE, Agrawal A. Metastatic carcinoma of the neck of unknown primary origin: evolution and efficacy of the modern workup. Arch Otolaryngol Head Neck Surg. 2009;135(10):1024-9.

31. Hosni A, Dixon PR, Rishi A, Au M, Xu W, Song Y, et al. Radiotherapy characteristics and outcomes for head and neck carcinoma of unknown primary vs T1 base-of-tongue carcinoma. JAMA Otolaryngol Head Neck Surg. 2016;142(12):1208-15

32. Nieder C, Gregoire V, Ang KK. Cervical lymph node metastases from occult squamous cell carcinoma: cut down a tree to get an apple? Int J Radiat Oncol Biol Phys. 2001;50(3):727-33.

33. Liu X, Li D, Li N, Zhu X. Optimization of radiotherapy for neck carcinoma metastasis from unknown primary sites: a meta-analysis. Oncotarget. 2016; 7(48):78736-46.

34. Gupta T, Agarwal J, Jain S, Phurailatpam R, Kannan S, Ghosh-Laskar S, et al. Three-dimensional conformal radiotherapy (3D-CRT) versus intensity modulated radiation therapy (IMRT) in squamous cell carcinoma of the head and neck: a randomized controlled trial. Radiother Oncol. 2012;104(3):343-8.

35. Ghosh-Laskar S, Yathiraj PH, Dutta D, Rangarajan V, Purandare N, Gupta T, et al. Prospective randomized controlled trial to compare 3-dimensional conformal radiotherapy to intensity-modulated radiotherapy in head and neck squamous cell carcinoma: long-term results. Head Neck. 2016;38(Suppl 1):E1481-7.

36. Bibault JE, Dussart $\mathrm{S}$, Pommier $\mathrm{P}$, Morelle $\mathrm{M}$, Huquet $\mathrm{M}$, Boisselier $\mathrm{P}$, et al Clinical outcomes of several IMRT techniques for patients with head and neck Cancer: a propensity score-weighted analysis. Int J Radiat Oncol Biol Phys. 2017;99(4):929-37.

37. Tribius S, Hoffmann AS, Bastrop S, Gorogh T, Haag J, Rocken C, et al. HPV status in patients with head and neck of carcinoma of unknown primary site: HPV, tobacco smoking, and outcome. Oral Oncol. 2012;48(11):1178-84.

38. Wallace A, Richards GM, Harari PM, Kirwan JM, Morris CG, Katakam H, et al. Head and neck squamous cell carcinoma from an unknown primary site. Am J Otolaryngol. 2011;32(4):286-90.

39. Dixon PR, Au M, Hosni A, Perez-Ordonez B, Weinreb I, Xu W, et al. Impact of p16 expression, nodal status, and smoking on oncologic outcomes of patients with head and neck unknown primary squamous cell carcinoma. Head Neck. 2016;38(9):1347-53.

40. Chen AM, Farwell DG, Lau DH, Li BQ, Luu Q, Donald PJ. Radiation therapy in the management of head-and-neck cancer of unknown primary origin: how does the addition of concurrent chemotherapy affect the therapeutic ratio? Int J Radiat Oncol Biol Phys. 2011;81(2):346-52.

41. Klem ML, Mechalakos JG, Wolden SL, Zelefsky MJ, Singh B, Kraus D, et al. Intensity-modulated radiotherapy for head and neck cancer of unknown primary: toxicity and preliminary efficacy. Int J Radiat Oncol Biol Phys. 2008; 70(4):1100-7.

42. Cooper JS, Pajak TF, Forastiere AA, Jacobs J, Campbell BH, Saxman SB, et al. Postoperative concurrent radiotherapy and chemotherapy for high-risk squamous-cell carcinoma of the head and neck. N Engl J Med. 2004;350(19): 1937-44.

43. Bernier J, Cooper JS, Pajak TF, van Glabbeke M, Bourhis J, Forastiere A, et al. Defining risk levels in locally advanced head and neck cancers: a comparative analysis of concurrent postoperative radiation plus chemotherapy trials of the EORTC (\#22931) and RTOG (\# 9501). Head Neck. 2005;27(10):843-50.
44. Bernier J, Domenge C, Ozsahin M, Matuszewska K, Lefèbvre J-L, Greiner RH, et al. Postoperative irradiation with or without concomitant chemotherapy for locally advanced head and neck Cancer. N Engl J Med. 2004;350(19): 1945-52.

45. Nguyen TK, Nguyen EK, Warner A, Louie AV, Palma DA. Failed Randomized Clinical Trials in Radiation Oncology: What Can We Learn? Int J Radiation Oncol Biol Phys. 2018;101(5):1018-24.

\section{Publisher's Note}

Springer Nature remains neutral with regard to jurisdictional claims in published maps and institutional affiliations.
Ready to submit your research? Choose BMC and benefit from:

- fast, convenient online submission

- thorough peer review by experienced researchers in your field

- rapid publication on acceptance

- support for research data, including large and complex data types

- gold Open Access which fosters wider collaboration and increased citations

- maximum visibility for your research: over $100 \mathrm{M}$ website views per year

At BMC, research is always in progress.

Learn more biomedcentral.com/submissions 